\title{
Charge relaxation resistance in the cotunneling regime of multi-channel Coulomb blockade: Violation of Korringa-Shiba relation
}

\author{
I. S. Burmistrov ${ }^{1,2}$ and Ya. I. Rodionov ${ }^{3}$ \\ ${ }^{1}$ L.D. Landau Institute for Theoretical Physics RAS, Kosygina street 2, 119334 Moscow, Russia \\ ${ }^{2}$ Moscow Institute of Physics and Technology, 141700 Moscow, Russia \\ ${ }^{3}$ Institute for Theoretical and Applied Electrodynamics RAS, Izhorskaya Str. 13, 125412 Moscow, Russia
}

(Dated: June 4, 2022)

\begin{abstract}
We study the low frequency admittance of a small metallic island coupled to a gate electrode and to a massive reservoir via a multi channel tunnel junction. The ac current is caused by a slowly oscillating gate voltage. We focus on the regime of inelastic cotunneling in which the dissipation of energy (the real part of the admittance) is determined by two-electron tunneling with creation of electron-hole pairs on the island. We demonstrate that at finite temperatures but low frequencies the energy dissipation is ohmic whereas at zero temperature it is super-ohmic. We find that (i) the charge relaxation resistance (extracted from the real part of the admittance) is strongly temperature dependent, (ii) the imaginary and real parts of the admittance do not satisfy the Korringa-Shiba relation. At zero temperature the charge relaxation resistance vanishes in agreement with the recent zero temperature analysis [M. Filippone and C. Mora, Phys. Rev. B 86, 125311 (2012) and P. Dutt, T. L. Schmidt, C. Mora, and K. Le Hur, Phys. Rev. B 87, 155134 (2013)].

PACS numbers: 73.23.Hk, 73.43.-f, 73.43.Nq
\end{abstract}

\section{INTRODUCTION}

During the last decades Coulomb blockade has become a powerful tool for observation of interaction and quantum effects in single electron devices [1-6]. This phenomenon is widely observed in low temperature electron transport through a single electron transistor. Another system which low temperature properties are affected by Coulomb blockade is a single electron box (SEB). It is schematically shown in Fig. 11. Small metallic island is coupled capacitively to the gate electrode with the voltage $U_{g}$. The number of electrons on the island is not conserved due to the tunneling in and out of an equilibrium electron reservoir. A time dependent gate voltage $U_{g}(t)$ generates ac current through the device.

The equivalent electric circuit of a SEB (see Fig. 1) is characterized by two capacitances. The gate capacitance $C_{g}$ controls the external (induced) charge $q$ on the island, $q=C_{g} U_{g}$. The total capacitance $C$ determines the so-called charging energy $E_{c}=e^{2} / 2 C$. It is the latter that is responsible for the Coulomb blockade effects. The tunnel junction is characterized by the dimensionless (in units $e^{2} / h$ ) conductance $g$. Throughout the paper we use a standard assumption that the Thouless energy of the island is the largest energy scale in the problem. This allows us to work in a zero dimensional approximation neglecting spatial dependence of all quantities.

Since there is no dc transport through the SEB, an essential dynamic characteristic becomes the admittance which characterizes the response of ac current $I_{\omega}$ to the infinitely small ac part $U_{\omega}$ of the time dependent gate voltage $U_{g}(t)=U_{0}+U_{\omega} \cos \omega t: \mathcal{G}(\omega)=I_{\omega} / U_{\omega}$. Long ago it was demonstrated that the admittance of SEB is affected by Coulomb blockade at low temperatures $T \ll E_{c}$ [7]. However, since then the majority of works have addressed the so-called quantum capaci- tance: $C_{\text {eff }}=\partial Q / \partial U_{g}$, where $Q$ is the average charge on the island, which determines the imaginary part of the admittance [8 14]. Classically, at high temperatures $T \gg E_{c}$ the effective capacitance coincides with $C_{g}$. As temperature decreases $C_{\text {eff }}$ starts to deviate from $C_{g}$ due to interaction and coherence effects. In seminal paper [15. it was suggested that the real and imaginary parts of the admittance in a SEB can be related in an universal way. After paper [15] a SEB admittance have attracted significant theoretical interest [16-20]. The admittance in the quasi-static regime was measured in a single channel SEB constructed in 2D electron gas 21. At present, there exists a number of measurements of admittances for different realizations of a SEB performed with the help of radio-frequency reflectometry 22,25$]$.

The classical electrodynamics of a SEB suggests the following expression for the admittance at low frequencies, $\omega \ll g E_{c}$ :

$$
\mathcal{G}(\omega)=-i \omega C_{g}+\omega^{2} C_{g} C R
$$

where $R=h /\left(e^{2} g\right)$ stands for the classical resistance of the tunnel junction. In Ref. 15 the following generalization of the classical result (1) has been proposed for
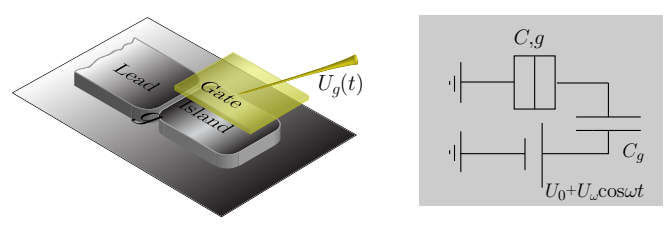

FIG. 1. (Color online) The set-up: a SEB subjected to a time-dependent gate voltage $U_{g}(t)$ (left) and the equivalent electric circuit (right). 
the quantum coherent SEB with $C=C_{g}$ :

$$
\mathcal{G}(\omega)=-i \omega C_{\mathrm{eff}}+\omega^{2} C_{\mathrm{eff}}^{2} R_{q},
$$

where $R_{q}$ was termed as charge relaxation resistance. Treating the Coulomb interaction within the HartreeFock approximation, the authors of Ref. [15] demonstrate that for single channel tunnel junction the charge relaxation resistance in Eq. (2) becomes universal, $R_{q}=$ $h /\left(2 e^{2}\right)$. The full quantum mechanical treatment of the charging energy in the case of single channel tunnel junction demonstrates that at zero temperature $R_{q}=h /\left(2 e^{2}\right)$ $\left(R_{q}=h / e^{2}\right)$ for frequencies $\omega \ll \delta(\omega \gg \delta)$ [26]. Here $\delta$ denotes the mean level spacing of single particle states inside the island of a SEB. Both results follow from two observations: (i) the effective low energy Hamiltonian of single channel SEB is of Fermi liquid type; (ii) the Korringa-Shiba relation 27/28 for the response function $i \mathcal{G}(\omega) / \omega$ holds within Fermi liquid low-energy description [26]. Recently, the analysis of Ref. 26] has been generalized to the case of a SEB with a weak $(g \ll 1)$ multi channel tunnel junction and a large island, $\delta \rightarrow 0$. It was found 2930 , that at zero temperature the charge relaxation resistance is inversely proportional to the number of channels in a tunnel junction and is independent of the external charge; $R_{q}$ vanishes in the limit of infinite number of channels for any value of $q$.

At finite temperatures the SEB with a multi channel tunnel junction in the limit of negligible mean level spacing, $\delta \rightarrow 0$, has been analyzed in Ref. 31. In particular, it was demonstrated that in the limit of weak tunneling, $g \ll 1$, and near the charge degeneracy points the SEB admittance at low frequencies $(\omega \ll g \max \{|\Delta|, T\})$ can be set down in the following form

$$
\mathcal{G}(\omega)=-i \omega C_{\mathrm{eff}}+\omega^{2} \frac{C}{C_{g}} \mathcal{C}_{g}^{2} \mathcal{R}_{q}
$$

Here $\Delta$ denotes the electrostatic energy due to one excess electron on the SEB island. It depends on the external charge $q$ and satisfy inequality $|\Delta| \ll E_{c}$ near a charge degeneracy point. The quantity $\mathcal{C}_{g}=\partial \mathcal{Q} / \partial U_{0}$ stands for the renormalized gate capacitance which measures the response of the effective charge $\mathcal{Q}$, introduced in Refs. 3233 by one of us, to the static part of the gate voltage. Contrary to the average charge $Q$ on the island, the effective charge is expected to be integer quantized at zero temperature 32 34. This implies that $\mathcal{C}_{g}$ vanishes at $T=0$ contrary to $C_{\text {eff }}$. The charge relaxation resistance in Eq. (3) is determined by the renormalized tunneling conductance $g(T), \mathcal{R}_{q}=h /\left(e^{2} g(T)\right) \gg h / e^{2}$. The very same conductance $g(T)$ determines the dc conductance of the single electron transistor (SET) under small bias between source and drain. We also note that Eq. (3) was proposed for the SEB with arbitrary relation between $C$ and $C_{g}$. For the case of weak tunneling the treatment of Ref. [31] was restricted to the sequential tunneling approximation dressed by the renormalization due to virtual processes. The processes of inelastic cotunneling 35] which dominate the dc transport through the
SET at low temperatures $T \ll T_{\text {in }} \sim|\Delta| / \ln (1 / g)$ were not taken into account. Therefore, the extrapolation of Eq. (3) down to the zero temperature and comparison with the result of Ref. 2930] were not possible.

The real part of the admittance of a SEB with a multi channel tunnel junction in the regime of inelastic cotunneling has been studied in Ref. [7]. It was found that the real part of admittance is proportional to $g^{2} \omega^{2} \max \left\{T^{4}, \omega^{4}\right\} / E_{c}^{2}$. This results implies the zero charge relaxation resistance at $T=0$ in agreement with the result of Ref. 2930 extrapolated to limit of the infinite number of channels in the tunnel junction. However, the analysis of Ref. [7] has been restricted to Coulomb valleys, i.e. to integer values of $q$.

In this paper we address the following question: how the zero temperature result for the charge relaxation resistance obtained in Refs. 2930] crosses over to the finite temperature result of Ref. [31]? To answer this question we performed a detailed study of the admittance of the multi channel SEB near the charge neutrality points in the low temperature regime where the inelastic cotunneling processes dominate the dynamics. We found that the real part of admittance is proportional to $g^{2} \omega^{2} \max \left\{T^{2}, \omega^{2}\right\} / \Delta^{4}$. The charge relaxation resistance (extracted from Eq. (2)) is strongly temperature dependent and small, $R_{q} \sim\left(h / e^{2}\right)(T / \Delta)^{2} \ll h / e^{2}$. In agreement with Refs. [2629], we obtained that $R_{q}$ is independent of $g$ and vanishes at zero temperature. Our explicit results demonstrate strong violation of Korringa-Shiba relation for the response function $i \mathcal{G}(\omega) / \omega$ and, consequently, support the non-Fermi liquid behavior of the multi channel SEB near the charge degeneracy points.

The structure of the paper is as follows. In Sec. II we introduce the Hamiltonian and Kubo formula for admittance of a single electron box. The pseudofermion representation for the low energy Hamiltonian valid in the cotunneling regime is presented in Sec. III. The results of calculation of the admittance at low frequencies to the second order in the tunneling conductance $g$ are given in Sec. IV. Finally, discussion of our results and conclusions are presented in Sec. V. The details of calculations are summarized in Appendix A. We use units with $\hbar=e=1$ through out the paper except for the final results.

\section{FORMALISM}

\section{A. Hamiltonian}

We start with the standard Hamiltonian describing Coulomb blockade in a SEB [36 38]:

$$
H=H_{l}+H_{d}+H_{c}+H_{t}
$$


Here $H_{l}\left(H_{d}\right)$ denotes free electron Hamiltonian in the lead (the island),

$$
\begin{aligned}
& H_{l}=\sum_{k} \varepsilon_{k}^{(a)} a_{k}^{\dagger} a_{k}, \\
& H_{d}=\sum_{\alpha} \varepsilon_{\alpha}^{(d)} d_{\alpha}^{\dagger} d_{\alpha},
\end{aligned}
$$

where operators $a_{k}^{\dagger}\left(d_{\alpha}^{\dagger}\right)$ create an electron in the reservoir (the island). Energies $\varepsilon_{k}^{(a)}, \varepsilon_{\alpha}^{(d)}$ are counted from the chemical potential. The Hamiltonian

$$
H_{c}=E_{c}\left(\hat{n}_{d}-q\right)^{2}, \quad \hat{n}_{d}=\sum_{\alpha} d_{\alpha}^{\dagger} d_{\alpha}
$$

takes into account the electrostatic energy due to the finite size of the island.

The Hamiltonian

$$
H_{t}=\sum_{k, \alpha} t_{k \alpha} a_{k}^{\dagger} d_{\alpha}+\text { h.c. }
$$

describes tunneling of electrons between the island and the reservoir. In order to characterize the tunnel junction, following Ref. [31, we introduce Hermitian matrix:

$$
\hat{g}_{\alpha \alpha^{\prime}}=(2 \pi)^{2}\left[\delta\left(\varepsilon_{\alpha}^{(d)}\right) \delta\left(\varepsilon_{\alpha^{\prime}}^{(d)}\right)\right]^{1 / 2} \sum_{k} t_{\alpha k}^{\dagger} \delta\left(\varepsilon_{k}^{(a)}\right) t_{k \alpha^{\prime}}
$$

acting in the Hilbert space of the island's states. Here the delta-functions are assumed to be smoothed on some intermediate scale between $\delta$ and $\min \{T,|\omega|\}$. The matrix $\hat{g}$ allows one to define the number of open channels $N_{\text {ch }}$ and the effective channel conductance $g_{\text {ch }}$ :

$$
N_{\text {ch }}=\frac{(\operatorname{tr} \hat{g})^{2}}{\operatorname{tr} \hat{g}^{2}}, \quad g_{\text {ch }}=\frac{\operatorname{tr} \hat{g}^{2}}{\operatorname{tr} \hat{g}} .
$$

The dimensionless conductance $g$ which characterizes the tunnel junction in classical electrodynamics is given as $g=g_{\mathrm{ch}} N_{\mathrm{ch}}$.

In the present paper we assume that $N_{\mathrm{ch}} \gg 1$ and $1 / N_{\text {ch }}^{2} \ll g_{\text {ch }} \ll 1$. Although within these assumptions the classical conductance $g$ can be still large, in what follows we restrict our consideration to the case $1 \gg g \gg$ $1 / N_{\mathrm{ch}}$. We are interested in temperatures much smaller than the charging energy but much larger than the mean level spacing, $E_{c} \gg T \gg \delta$.

\section{B. Admittance and polarization operator}

The admittance of a SEB being the linear response of the ac current to the ac part of the time-dependent gate voltage, $U_{g}(t)=U_{0}+U_{\omega} \cos \omega t$, can be expressed as 31.

$$
\mathcal{G}(\omega)=-i \omega C_{g}\left(1+\Pi^{R}(\omega) / C\right),
$$

where $\Pi^{R}(\omega)$ stands for the Fourier transform of the retarded polarization operator of electrons on the island:

$$
\Pi^{R}(t)=i \Theta(t)\left\langle\left[\hat{n}_{d}(t), \hat{n}_{d}(0)\right]\right\rangle .
$$

Here $\Theta(t)$ denotes the Heaviside step function. In the quasi-static regime $\omega \rightarrow 0$, the polarization operator $\Pi^{R}(\omega)$ can be expanded in regular series in $\omega$ :

$$
\Pi^{R}(\omega)=\pi_{0}+i \omega \pi_{1}+\mathcal{O}\left(\omega^{2}\right),
$$

where both $\pi_{0}$ and $\pi_{1}$ are real functions. We stress that we assume $|\omega| \gg \delta$ throughout the paper. The static part $\pi_{0}$ is fully determined by the average charge on the island $Q$ :

$$
\pi_{0}=\frac{C}{C_{g}} C_{\mathrm{eff}}-C
$$

where we remind $C_{\text {eff }}=\partial Q / \partial U_{0}$ with $Q=\left\langle\hat{n}_{d}\right\rangle$. This result holds by virtue of the Ward identity which relates the static polarization operator and the compressibility 39]. We note that on the classical level $C_{\text {eff }}=C_{g}$ and $\pi_{0}=0$. The classical electrodynamics result (1) implies $\pi_{1}=2 \pi C^{2} / g$ on the classical level. In what follows we discuss how quantum effects due to inelastic cotunneling change naive classical expectations for $\pi_{0}$ and $\pi_{1}$.

\section{WEAK TUNNELING REGIME}

\section{A. Projected Hamiltonian}

In what follows, contrary to Ref. [7, we confine our consideration to the vicinity of the one of charge degeneracy points, i.e. points where the external charge $q=k+1 / 2$ where $k$ is an integer. At these points the gap $\Delta=2 E_{c}(k+1 / 2-q)$ between the ground and the first excited state of the charging Hamiltonian $H_{c}$ vanishes. In the vicinity of the degeneracy point, the gap $\Delta$ is small in comparison with the charging energy, $|\Delta| \ll E_{c}$. The processes of inelastic cotunneling becomes important at low temperatures $T \ll|\Delta|$. At $|\Delta| \ll E_{c}$, one can truncate the Hilbert space of electrons on the isolated island to two charging states characterized by $Q=k$ and $Q=k+1$ [8]. The projected Hamiltonian acquires a form of $2 \times 2$ matrix acting in the isospin $1 / 2$ space of these two charging states [8]:

$$
\tilde{H}=H_{l}+H_{d}+\tilde{H}_{t}+\Delta S_{z}+\frac{\Delta^{2}}{4 E_{c}}+\frac{E_{c}}{4},
$$

where $H_{l, d}$ are given by Eq. (5), and

$$
\tilde{H}_{t}=\sum_{k, \alpha} t_{k \alpha} a_{k}^{\dagger} d_{\alpha} S^{+}+\text {h.c. . }
$$

Here $S^{z}, S^{ \pm}=S^{x} \pm i S^{y}$ stand for standard spin $1 / 2$ operators. The Hamiltonian (14) is the Hamiltonian for 
the $N_{\text {ch }}$ channel Kondo model. The gap $\Delta$ between the charging states plays the role of an effective magnetic field.

In the presence of ac component of the gate voltage, the energy detuning from the degeneracy point becomes time dependent: $\Delta(t)=\Delta-\left(C_{g} / C\right) U_{\omega} \cos \omega t$. Then, as follows from Eq. (14), the linear response of a SEB to ac gate voltage $U_{\omega}$ is determined by the longitudinal dynamical isospin susceptibility:

$$
\Pi_{s}^{R}(t)=i \Theta(t)\left\langle\left[S_{z}(t), S_{z}(0)\right]\right\rangle .
$$

In particular, the admittance $\mathcal{G}(\omega)$ is given as [31]:

$$
\mathcal{G}(\omega)=-i \omega \frac{C_{g}}{C} \Pi_{s}^{R}(\omega)
$$

We note that the average charge on the island can be written as $Q=k+1 / 2-\left\langle S_{z}\right\rangle$, i.e. it is related to the isospin magnetization. As the consequence of Eq. (13), the dynamical isospin susceptibility should satisfy the relation $\Pi_{s}^{R}(0)=C C_{\text {eff }} / C_{g}$.

\section{B. Pseudofermion effective action}

To deal with spin operators we employ the method of Abrikosov's pseudofermion operators. Following Ref. [40, we introduce fermion operators $\bar{\psi}_{\alpha}, \psi_{\alpha}$ such that

$$
\boldsymbol{S}=\frac{1}{2} \bar{\psi}_{\alpha} \boldsymbol{\sigma}_{\alpha \beta} \psi_{\beta}
$$

Here $\boldsymbol{\sigma}=\left\{\sigma_{x}, \sigma_{y}, \sigma_{z}\right\}$ denotes standard Pauli matrices. To exclude redundant unphysical states (the states with $\sum_{\alpha} \bar{\psi}_{\alpha} \psi_{\alpha}>1$ ) we add to the Hamiltonian $\tilde{H}$ an artificial chemical potential $\eta$ for the pseudofermions. It is necessary to take the limit $\eta \rightarrow-\infty$ at the end of any calculation.

We remind that the physical partition function $\mathcal{Z}$ and correlation functions $\langle\mathcal{O}\rangle$ can be found from the pseudofermion ones with the help of the following rules:

$$
\begin{aligned}
\mathcal{Z} & =\lim _{\eta \rightarrow-\infty} \frac{\partial}{\partial e^{\beta \eta}} \mathcal{Z}_{\mathrm{pf}}, \\
\langle\mathcal{O}\rangle & =\lim _{\eta \rightarrow-\infty}\left\{\langle\mathcal{O}\rangle_{\mathrm{pf}}+\frac{\mathcal{Z}_{\mathrm{pf}}}{\mathcal{Z}} \frac{\partial}{\partial e^{\beta \eta}}\langle\mathcal{O}\rangle_{\mathrm{pf}}\right\} .
\end{aligned}
$$

In the case $N_{\text {ch }} \gg 1$ and $1 \gg g \gg 1 / N_{\text {ch }}$, after the integration over electrons in the reservoir and the island the Hamiltonian (14) can be transformed into the following imaginary-time effective action [31]:

$$
\begin{aligned}
S & =\frac{\beta \Delta^{2}}{4 E_{c}}+\int_{0}^{\beta} d \tau \bar{\psi}\left(\partial_{\tau}+\frac{\sigma_{z} \Delta}{2}-\eta\right) \psi \\
& +\frac{g}{4} \int_{0}^{\beta} d \tau_{1} d \tau_{2} \alpha\left(\tau_{12}\right)\left[\bar{\psi}\left(\tau_{1}\right) \sigma_{-} \psi\left(\tau_{1}\right)\right]\left[\bar{\psi}\left(\tau_{2}\right) \sigma_{+} \psi\left(\tau_{2}\right)\right]
\end{aligned}
$$

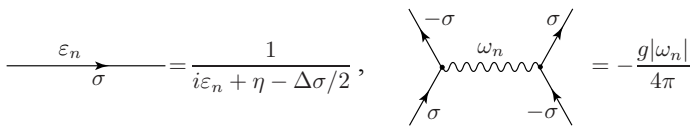

FIG. 2. Feynman rules for the pseudofermion action 20].

Here $\sigma_{ \pm}=\left(\sigma_{x} \pm i \sigma_{y}\right) / 2$ and the kernel

$$
\alpha(\tau)=\frac{T}{\pi} \sum_{\omega_{n}}\left|\omega_{n}\right| e^{-i \omega_{n} \tau},
$$

where $\omega_{n}=2 \pi T n$ is the bosonic Matsubara frequency. We note that the action similar to Eq. (20) has been first analyzed by Larkin and Melnikov in Ref. 41. Effective action 20 corresponds to the XY Bose-Kondo model for the spin 1/2 42,4344].

The dynamical spin susceptibility 16 is determined by the pseudofermion dynamical spin susceptibility:

$$
\Pi_{s, \mathrm{pf}}(\tau)=\frac{1}{4}\left\langle\mathcal{T}_{\tau}\left[\bar{\psi}(\tau) \sigma_{z} \psi(\tau)\right]\left[\bar{\psi}(0) \sigma_{z} \psi(0)\right]\right\rangle,
$$

where $\mathcal{T}_{\tau}$ denotes the imaginary-time ordering and the average is taken with respect to the effective action 20 . According to Eq. 19 we also need the expression for the physical partition function. It can be written as

$$
\mathcal{Z}=\left.\lim _{\eta \rightarrow 0} \mathcal{Z}_{\mathrm{pf}} e^{-\beta \eta} \sum_{\sigma} \mathcal{G}_{\sigma}(\tau)\right|_{\tau \rightarrow 0^{-}},
$$

where $\mathcal{G}_{\sigma}(\tau)=-\left\langle\mathcal{T}_{\tau} \psi_{\sigma}(\tau) \bar{\psi}_{\sigma}(0)\right\rangle$ stands for the exact imaginary-time pseudofermion Green's function.

As we discussed in the Introduction, in the Fermi liquid the imaginary and real parts of the dynamical spin susceptibility are related by the so-called Korringa-Shiba relation 2728 . Taking into account that $\Delta$ in the effective action 20 plays a role of magnetic field, KorringShiba relation for $\Pi_{s}^{R}$ should have the following universal form:

$$
\operatorname{Im} \Pi_{s}^{R}(\omega) \stackrel{?}{=} 2 \pi \omega\left[\operatorname{Re} \Pi_{s}^{R}(0)\right]^{2}, \quad \omega \rightarrow 0 .
$$

If this relation were correct, the low-frequency admittance $\mathcal{G}(\omega)=-i \omega\left(C_{g} / C\right)\left(C_{0}+i \omega C_{0}^{2} R_{q}\right)$ would be characterized by the universal charge relaxation resistance, $R_{q}=h / e^{2}$, similar to the single channel case. Here we introduce $C_{0}=\left(C / C_{g}\right) C_{\text {eff }}$. As we shall demonstrate below by direct calculation, Korringa-Shiba relation 24 does not hold for the effective action 20 .

\section{ADMITTANCE IN THE COTUNNELING REGIME}

The effective action 20 is suitable for the perturbation theory in $g \ll 1$. Here we evaluate the imaginary part of the dynamical spin susceptibility $\Pi_{s}(\omega)$ to the second order in $g$. The Feynman rules for action 20 

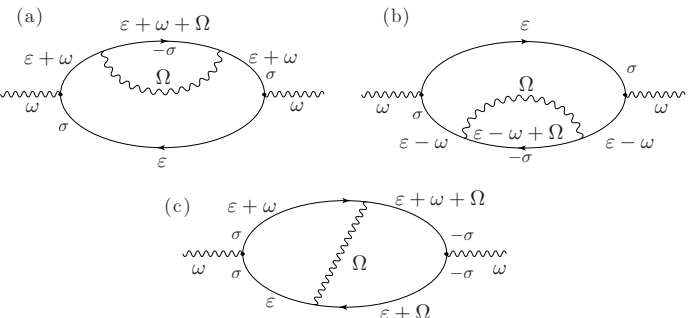

FIG. 3. Feynman diagrams for the pseudofermion dynamical spin susceptibility in the first order in $g$.

are shown in Fig. 2. The bare pseudofermion Matsubara Green's function is given as follows:

$$
G_{\sigma}\left(i \varepsilon_{n}\right)=\frac{1}{i \varepsilon_{n}+\eta-\sigma \Delta / 2} .
$$

Thus from Eqs. 22 and 23 in the zeroth order in $g$ we find

$$
\mathcal{Z}_{\mathrm{pf}}^{(0)}=1, \quad \mathcal{Z}^{(0)}=2 \cosh \frac{\beta \Delta}{2}, \quad \Pi_{s, \mathrm{pf}}^{(0)}\left(i \omega_{n}\right)=0 .
$$

\section{A. Perturbation theory in $g$ : Sequential tunneling}

Before discussing the inelastic cotunneling regime (second order in $g$ ) we remind briefly the result of Ref. 31. for the pseudofermion dynamical spin susceptibility in the regime of sequential tunneling. In Fig. 3 we present diagrams contributing to the pseudofermion dynamical spin susceptibility $\Pi_{s, \text { pf }}\left(i \omega_{n}\right)$ in the first order in $g$. Their evaluation demonstrates that $\operatorname{Im}_{\Pi_{s}^{R}}^{R}(\omega)$ suffers from singularity at $\omega \rightarrow 0$ :

$$
\operatorname{Im} \Pi_{s}^{R,(1)}(\omega)=\frac{g}{4 \pi \omega} \frac{\beta \Delta}{\sinh (\beta \Delta)} .
$$

This unphysical divergence stems from noncommutativity of the limits $\omega \rightarrow 0$ and $g \rightarrow 0$ in the structure of the $\operatorname{Im} \Pi_{s}^{R,(1)}(\omega)$. Summing the laddertype diagrams and taking into account finite (the lowest order in $g$ ) broadening of the pseudofermion Green's function, we obtain the following expression [31]:

$$
\Pi_{s}^{R,(\mathrm{lad})}(\omega)=\frac{g}{4 \pi} \frac{\beta \Delta}{\sinh (\beta \Delta)}\left(-i \omega+\frac{g \Delta}{2 \pi} \operatorname{coth} \frac{\beta \Delta}{2}\right)^{-1}
$$

We note that the broadening of the singularity at $\omega=0$ in $\operatorname{Im} \Pi_{s, \mathrm{pf}}^{R}(\omega)$ is determined by the sum of in- and outrate of single electron tunneling [3]. At low temperatures $T \ll|\Delta|$, the result 28 implies $(|\omega| \ll g|\Delta| / 2 \pi)$ :

$$
\operatorname{Im} \Pi_{s}^{R,(\operatorname{lad})}(\omega)=\frac{2 \pi \beta \omega}{g|\Delta|} e^{-\beta|\Delta|} .
$$

Such Arrhenius-type dependence, $\exp (-|\Delta| / T)$, is characteristic of real processes in which an additional electron or hole remains on the island after each tunneling event. We remind that the SET conductance in the sequential tunneling approximation is also of the same Arrheniustype form at low temperatures $T \ll|\Delta| 331$. As it is well-known, at low temperatures SET conductance has also a power-law (in $T /|\Delta|$ ) contribution of the second order in $g$ due to the processes of inelastic cotunneling 35. As we shall demonstrate in the next section there is a similar contribution to the admittance.

\section{B. Perturbation theory in $g$ : Inelastic cotunneling}

The processes of inelastic cotunneling becomes important at temperatures $T \ll T_{\text {in }} \ll|\Delta|$. However, these processes are of the second order in the tunneling conductance. Diagrams of the second order in $g$ for the pseudofermion dynamical spin susceptibility are shown in Fig. 4. We remind that diagrams with pseudofermion loops vanish in the limit $\eta \rightarrow-\infty$. Evaluation of the ten diagrams in Fig. 4 yields the following result for the imaginary part of the low-frequency dynamical spin susceptibility (see Appendix A)

$$
\operatorname{Im} \Pi_{s}^{R,(2)}(\omega)=\frac{g^{2} \omega}{24 \pi \Delta^{4}}\left(T^{2}+\frac{\omega^{2}}{4 \pi^{2}}\right), \quad|\omega|, T \ll|\Delta| .
$$

This expression dominates over the contribution $(29)$ due to sequential tunneling at low temperatures, $T \ll T_{\text {in }}$.

In the discussion above we do not consider renormalization of the effective action $(20)$ between the ultra violet energy scale of the order of $E_{c}$ and the infrared scale of the order of $\max \{|\Delta|, T\}$ [1/8,45]. The renormalized effective action can be obtained from Eq. (20) by the following substitutions $\psi \rightarrow \sqrt{Z} \psi, \bar{\psi} \rightarrow \sqrt{Z} \psi, g \rightarrow \bar{g}=Z^{2} g$ and $\Delta \rightarrow \bar{\Delta}=Z^{2} \Delta$, where the field renormalization factor $Z$ is given within one-loop approximation as [4]

$$
Z=\left[1+\frac{g}{2 \pi^{2}} \ln \frac{E_{c}}{\max \{|\bar{\Delta}|, T\}}\right]^{-1 / 2} .
$$

We note that the pseudospin operator $S_{z}$ renormalizes according to $S_{z} \rightarrow Z^{2} S_{z}$ [31. Then from Eq. (30) we find

$$
\operatorname{Im} \Pi_{s}^{R,(2)}(\omega)=\frac{Z^{4} \bar{g}^{2} \omega}{24 \pi \bar{\Delta}^{4}}\left(T^{2}+\frac{\omega^{2}}{4 \pi^{2}}\right) .
$$

Remarkably, the field renormalization parameter $Z$ drops out from Eq. (32) such that it coincides with Eq. (30) in spite of the renormalization.

Taking into account one-loop renormalization of the effective action, one finds the following result for the average charge on the island [8]:

$$
Q=k+\frac{1}{2}-\frac{Z^{2}}{2} \tanh \frac{\bar{\Delta}}{2 T} .
$$


I
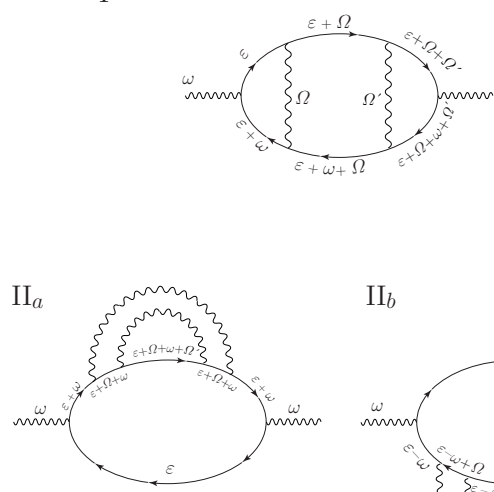

$\mathrm{II}_{b}$

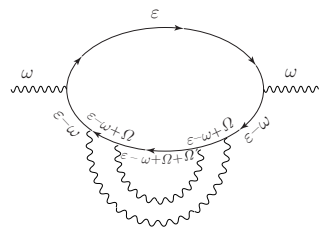

$\mathrm{III}_{a}$

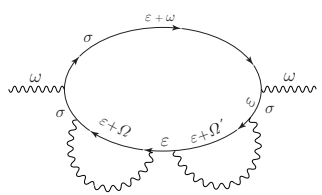

$\mathrm{III}_{b}$
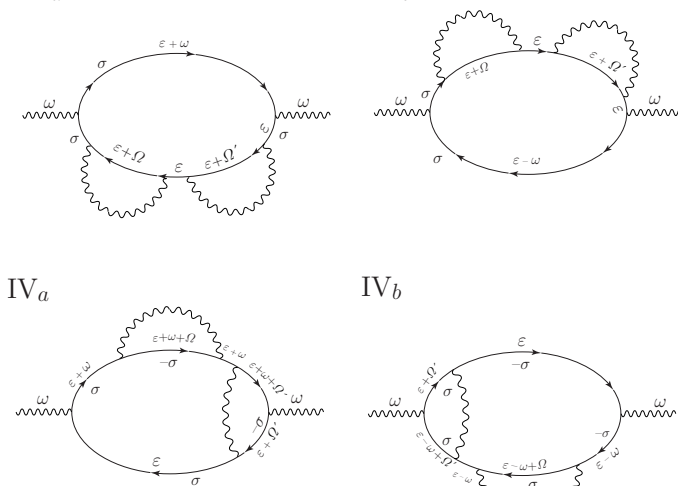

$\mathrm{IV}_{b}$
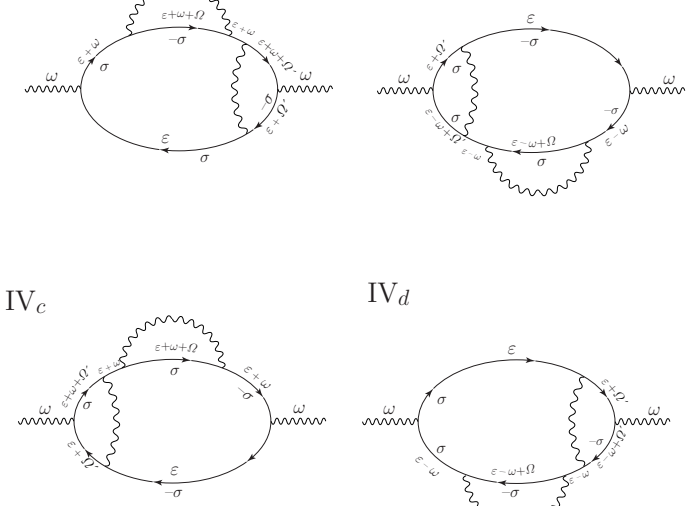

$\mathrm{IV}_{d}$
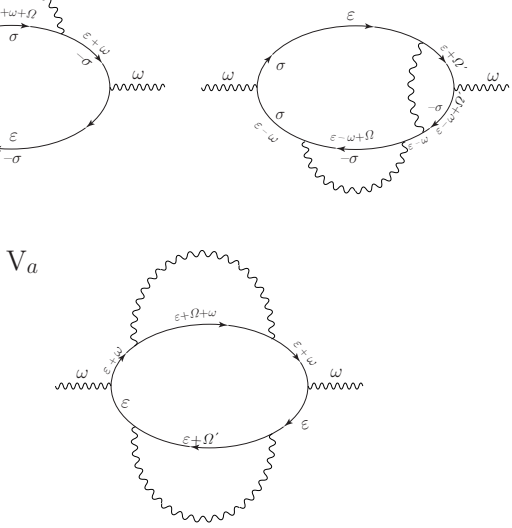

FIG. 4. Second order in $g$ diagrams for the pseudofermion dynamical spin susceptibility.

In the case of low temperatures, $T \ll T_{\text {in }}$, Eq. 33 can be simplified, and we obtain

$$
\operatorname{Re} \Pi_{s}^{R,(1)}(0)=-\frac{\partial Q}{\partial \Delta}=\frac{Z^{4} \bar{g}}{4 \pi^{2}|\bar{\Delta}|} .
$$

We note that the factor $Z^{4}$ can be derived in the following way. Equation (31) determines the one-loop renormalization group equation for the field renormalization factor. Then taking into account that (i) the relation $\partial \bar{\Delta} / \partial \Delta=Z^{2}$ holds within logarithmic accuracy and (ii) the renormalized conductance $\bar{g}$ corresponds to the energy scale $\bar{\Delta}$, one can obtain the result (34). Alternatively, the appearance of the factor $Z^{4}$ can be checked with the help of the expression for $Q$ derived to the second order in $g$ 33. We emphasize that Eq. (34) implies that at $T \ll T_{\text {in }}$ the effective capacitance becomes very different from $C_{g}: C_{\text {eff }}=C_{g} Z^{4} \bar{g} E_{c} /\left(2 \pi^{2}|\bar{\Delta}|\right)$.

Combining together Eqs. (30) and (34) we obtain the following result for the admittance of a SEB $(|\omega| \ll T \ll$ $\left.T_{\text {in }}\right)$ :

$$
\mathcal{G}(\omega)=-i \omega C_{g} \frac{Z^{4} \bar{g} E_{c}}{2 \pi^{2}|\bar{\Delta}|}+\omega^{2} C_{g} \frac{Z^{4} \bar{g}^{2} T^{2} E_{c}}{12 \pi \bar{\Delta}^{4}} .
$$

This is the main result of the present paper. Results (32) and (34) for the imaginary and real part of the dynamical isospin susceptibility implies strong violation of the Korringa-Shiba relation (24) at low temperatures when the processes of inelastic cotunneling dominate. Using the Korringa-Shiba relation, one overestimates erroneously $\operatorname{Im} \Pi_{s}^{R}(\omega)$ by a large factor $(\bar{\Delta} / T)^{2} \gg 1$. The violation of the Korringa-Shiba relation signals that the Hamiltonian (14) and, consequently, the effective action 20 , involves non-Fermi liquid physics.

\section{DISCUSSION AND CONCLUSIONS}

According to Eq. (2), the result (35) for the admittance implies the following result for the charge relaxation resistance:

$$
R_{q}=\frac{h}{e^{2}} \frac{\pi^{2}}{3}\left(\frac{T}{Z^{2} \bar{\Delta}}\right)^{2}, \quad T \ll T_{\mathrm{in}} \sim \frac{|\bar{\Delta}|}{\ln (1 / \bar{g})} .
$$

We emphasize that due to Coulomb interaction the charge relaxation resistance is strongly temperature and gate voltage dependent. Moreover, $R_{q}$ depends on the tunneling conductance $g$ although through the field renormalization factor only. Therefore, the charge relaxation resistance depends in nontrivial way on the parameters of a SEB in contrast to the zero temperature predictions of Refs. 29 30] and original ideas of Ref. 15. Also the charge relaxation resistance is much smaller than the resistance quantum, $R_{q} \ll h / e^{2}$. At $T=0$ the charge relaxation resistance vanishes, $R_{q}=0$. This behavior is in agreement with the zero temperature result of Refs. 29.30, $R_{q}(T=0)=\left(h / e^{2}\right) / N_{\mathrm{ch}}$, which implies zero charge relaxation resistance at $T=0$ and $N_{\mathrm{ch}} \rightarrow \infty$.

The real part of the admittance determines the energy dissipation rate of a $\mathrm{SEB}, \mathcal{W}=C_{g} E_{c} \operatorname{Re} \mathcal{G}(\omega) U_{\omega}^{2}$, which appears due to the time dependent periodic gate voltage modulations. The result (35) leads to the ohmic dissipation at low frequencies:

$$
\mathcal{W}=\omega^{2} \frac{Z^{4} \bar{g}^{2} T^{2} E_{c}^{2}}{3 \pi^{2} \bar{\Delta}^{4}} C_{g}^{2} U_{\omega}^{2}, \quad|\omega| \ll T \ll T_{\mathrm{in}} .
$$


(a)
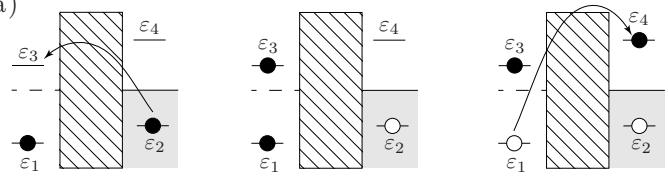

(b)
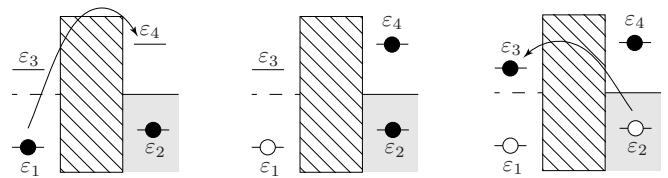

FIG. 5. The processes of inelastic cotunneling. The intermediate state has an additional electron (a) and hole (b).

This result for the dissipation rate has the following physical explanation. Let us estimate the rate $\Gamma_{\text {in }}$ for the two-electron process in which one electron with energy $\varepsilon_{1}$ tunnels from the island into the reservoir and occupies the state with energy $\varepsilon_{4}$ whereas the other electron with energy $\varepsilon_{2}$ tunnels from the reservoir into the island occupying the state with energy $\varepsilon_{3}$ (see Fig. 5). For $\varepsilon_{1} \neq \varepsilon_{3}$ this process is inelastic and results in the electron-hole pair on the island at the end. It can go through two different intermediate states: with an additional electron and an additional hole on the island. The former costs the energy of the order of $2 E_{c}$ whereas the latter costs the energy of the order of $|\Delta|$. In the considered case $|\Delta| \ll E_{c}$, we can neglect the contribution due to the intermediate state with an additional electron. Provided such transition is accompanied by a periodic perturbation which supplies the energy $\omega$ to the final state, we can estimate the corresponding rate as follows

$$
\begin{aligned}
\Gamma_{\mathrm{in}} \sim & \frac{g^{2}}{\Delta^{2}}\left(\prod_{j=1}^{4} \int_{-\infty}^{\infty} d \varepsilon_{j}\right) f_{F}\left(\varepsilon_{1}\right)\left[1-f_{F}\left(\varepsilon_{3}\right)\right] f_{F}\left(\varepsilon_{2}\right) \\
& \times\left[1-f_{F}\left(\varepsilon_{4}\right)\right] \delta\left(\varepsilon_{3}+\varepsilon_{4}-\varepsilon_{1}-\varepsilon_{2}-\omega\right) .
\end{aligned}
$$

Here we use the fact that typical electron or hole energies in the integral in Eq. (38) are of the order of $\max \{T,|\omega|\} \ll|\Delta|$. Then for $|\omega| \ll T \ll|\Delta|$ we find that the frequency dependent part of the rate is estimated as $\Gamma_{\text {in }}^{(\omega)} \sim g^{2} T^{2} \omega / \Delta^{2}$. In fact, this rate is similar to the rate derived for a SET biased by voltage in which case the role of $\omega$ is played by dc voltage 35 . The quadratic dependence of $\Gamma_{\text {in }}^{(\omega)}$ on $T$ is responsible for the $T^{2}$ factor in the expression (37) for the energy dissipation rate. Indeed, averaging $\Gamma_{\text {in }}^{(\omega)}$ over time-dependent gate voltage, taking contribution proportional to $U_{\omega}^{2}$ and multiplying the result by $\omega$, one gets the estimate for the rate of energy dissipation, $\mathcal{W} \sim \omega\left(\partial^{2} \Gamma_{\text {in }}^{(\omega)} / \partial \Delta^{2}\right)\left(C_{g} U_{\omega} / C\right)^{2}$, which, up to numerical factors, coincides with the result (37). At zero temperature the rate of inelastic cotunneling is given as $\Gamma_{\text {in }} \sim g^{2} \omega^{3} / \Delta^{2}$. This suggests the following estimate for the energy dissipation rate $\mathcal{W} \sim \omega^{4} g^{2} E_{c}^{2} C_{g} U_{\omega} / \Delta^{4}$, i.e. non-ohmic dissipation of energy at zero temperature. Using the result 32 with $T=0$, we obtain the following expression for the energy dissipation rate at zero temperature:

$$
\mathcal{W}=\frac{Z^{4} \bar{g}^{2} \omega^{4} E_{c}^{2}}{12 \pi^{4} \bar{\Delta}^{4}} C_{g}^{2} U_{\omega}^{2}, \quad T \ll|\omega| \ll|\bar{\Delta}| .
$$

It is worthwhile to discuss the result of Ref. 7] which is complementary to the result of the present paper. In Ref. 7] the real part of admittance was analyzed in the regime of inelastic cotunneling but precisely at $q=k$, i.e. at Coulomb valleys. It was found that $\operatorname{Re} \mathcal{G}(\omega) \propto g^{2} \omega^{2} \max \left\{T^{4}, \omega^{4}\right\} / E_{c}^{6}$. We emphasize that the result of Ref. [7] is quite unexpected. For example, the contribution due to inelastic cotunneling is proportional to $T^{2} / \Delta^{2}$ near the charge neutrality points and to $T^{2} / E_{c}^{2}$ at Coulomb valleys 35 . Therefore, on the basis of our result one could expect that at Coulomb valleys the energy dissipation rate is given by Eqs. (37) and (39) with $\Delta$ substituted by $E_{c}$. However, this naïve argument leads to overestimation of the energy dissipation rate by large factor $E_{c}^{2} / \max \left\{T^{2}, \omega^{2}\right\}$. The result of Ref. [7] comes from particular cancellation of terms proportional to $\omega^{2} T^{2} / E_{c}^{4}$ and $\omega^{4} / E_{c}^{4}$ in $\operatorname{Re} \mathcal{G}(\omega)$ (see comment at the end of Appendix A.

We mention that our result (35) is at odds with the expression (3) proposed by us in Ref. [31. Since the effective charge $\mathcal{Q}$ is expected to be robustly integer quantized at zero temperature 32,34 , the renormalized gate capacitance $\mathcal{C}_{g}$ is exponentially small at $T \ll|\bar{\Delta}|[33$. The renormalized conductance is known to be proportional to the temperature squared, $g(T) \sim \bar{g}^{2} T^{2} / \bar{\Delta}^{2}$, in the regime of the inelastic cotunneling [35/33. Therefore, Eq. (35) suggests the exponential suppression of the energy dissipation at $T \ll|\bar{\Delta}|$ contrary to the result (37). Thus for $g \ll 1$ the expression (3) works within the sequential tunneling approximation dressed by renormalization due to virtual processes only.

The effective action (20) predicts zero value of $\bar{g}$ under the renormalization in the infra red. The $N_{\text {ch }}$ channel Kondo model (14) has the unstable fixed point at finite value of $\bar{g}=g_{*} \sim 1 / N_{\text {ch }}$ 846]. Therefore, our results obtained within the effective action 20 are applicable for the Hamiltonian (14) while $\bar{g} \gg g_{*}$. As follows from Eq. (31), this condition implies that our results hold not too close to the charge degeneracy point, $|\Delta| \gg\left(g E_{c} / \pi^{2}\right) \exp \left(-\pi^{2} / g_{*}\right)$. Since $g_{*} \sim 1 / N_{\mathrm{ch}}$, the scale $\left(g E_{c} / \pi^{2}\right) \exp \left(-\pi^{2} / g_{*}\right)$ becomes extremely small already for not too very large values of $N_{\mathrm{ch}}$. Comparing our result (36) with the zero temperature result of Refs. 29.30, we find that for the case of finite number of channels the charge relaxation resistance is given by Eq. (36) for temperatures $T \gg|\bar{\Delta}| / \sqrt{N_{\mathrm{ch}}}$.

To summarize, we have studied the low frequency admittance of a multi channel SEB under a slowly oscillating gate voltage. Focusing on the regime of inelastic 
cotunneling, we have calculated the admittance $\mathcal{G}(\omega)$ (see Eq. (35p) near the charge degeneracy points. We found the following:

(i) At finite temperatures but low frequencies, $T_{\mathrm{in}} \gg$ $T \gg|\omega|$, the energy dissipation rate (determined by the real part of the admittance) is ohmic and scales as the temperature squared, see Eq. (37), in agreement with qualitative arguments.

(ii) At zero temperature the energy dissipation rate is super-ohmic, $\sim \omega^{4}$, see Eq. (39), in agreement with qualitative estimates.

(iii) The imaginary and real parts of the response function $i \mathcal{G}(\omega) / \omega$ do not satisfy Korringa-Shiba relation. This supports the non-Fermi liquid behavior of the model near the charge degeneracy points.

(iv) The charge relaxation resistance $R_{q}$ is strongly temperature dependent and small, $R_{q} \sim\left(h / e^{2}\right)(T / \bar{\Delta})^{2} \ll h / e^{2}$. It vanishes at $T=0$ in agreement with the recent zero temperature analysis of Refs. 2930 .

(v) The relation between the real part of admittance and the effective charge $\mathcal{Q}$ conjected by us in Ref. 31] does not hold beyond the sequential tunneling approximation dressed by renormalization due to virtual processes.

Finally, we mention that our result 35 for the admittance can be tested in a single electron box with small metallic island via radio-frequency reflectometry measurements 2225. Also we mention that following approach of Refs. [47/48] our results can be extended to non-equlibrium conditions, e.g. different temperatures of the island and the reservoir [49.

\section{ACKNOWLEDGMENTS}

We acknowledge useful discussions with A. Ioselevich, Yu. Makhlin, and, especially, with C. Mora and Yu. Nazarov. The research was funded by Russian Science Foundation under the grant No. 14-02-00898.

\section{Appendix A: Computation of the polarization operator: Diagrams of the second order in $g$}

In this appendix we present details of computation of the polarization operator within the second order perturbation theory in $g$. There are contributions from the ten diagrams shown in Fig. 4. The task is simplified considerably by the fact that we only need the imaginary part of the retarded polarization operator. Each diagram consists of six pseudofermion Green's function lines and two interaction lines. Thus each diagram involves the summation over three internal energies: fermionic $\varepsilon$ and two bosonic ones $\Omega, \Omega^{\prime}$. As usual the fermionic sum is easily undertaken with the help of the following identity: $T \sum_{\varepsilon} f(\varepsilon)=(4 \pi i)^{-1} \oint d \varepsilon \tanh (\varepsilon / 2 T) f(\varepsilon)$ where the contour of integration circles around all the poles of tanh.

\section{The diagram I}

The contribution from the diagram I to the polarization operator can be written as

$$
\begin{aligned}
& \Pi_{s, \mathrm{pf}}^{(2), \mathrm{I}}\left(i \omega_{n}\right)=-\frac{T^{3}}{4} \sum_{\sigma= \pm} \sigma^{2} \sum_{\varepsilon, \Omega, \Omega^{\prime}} \tilde{\alpha}(i \Omega) \tilde{\alpha}\left(i \Omega^{\prime}\right) G_{\sigma}(i \varepsilon) \\
& \times G_{\sigma}\left(i \varepsilon+i \omega_{n}\right) G_{-\sigma}(i \varepsilon+i \Omega) G_{-\sigma}\left(i \varepsilon+i \Omega+i \omega_{n}\right) \\
& \times G_{\sigma}\left(i \varepsilon+i \Omega+i \Omega^{\prime}\right) G_{\sigma}\left(i \varepsilon+i \Omega+i \Omega^{\prime}+i \omega_{n}\right),
\end{aligned}
$$

where we introduce the kernel $\tilde{\alpha}(i \Omega)=g \alpha(i \Omega) / 4=$ $g|\Omega| /(4 \pi)$. Evaluating the sum over the fermionic energy $\varepsilon$ and performing analytic continuation, $i \omega_{n} \rightarrow \omega+i 0$, we obtain

$$
\begin{gathered}
\operatorname{Im} \Pi_{s, \mathrm{pf}}^{R,(2), \mathrm{I}}(\omega)=-\frac{T}{4 \omega} \sum_{\sigma= \pm}\left(f_{\sigma}^{\prime}+f_{-\sigma}^{\prime}\right) \operatorname{Im} K_{\sigma}^{R,(2,1)}(\omega) \\
+\sum_{\sigma= \pm} \frac{f_{\sigma}-f_{-\sigma}}{2 \omega} \operatorname{Im}\left[K_{\sigma}^{R,(1,1)}(\omega)\right]^{2} .
\end{gathered}
$$

Here $f_{\sigma}=f_{F}(-\eta+\Delta \sigma / 2), f_{\sigma}^{\prime}=\partial f_{F}(\varepsilon) /\left.\partial \varepsilon\right|_{\varepsilon=-\eta+\Delta \sigma / 2}$ and $f_{F}(\varepsilon)=1 /[1+\exp (\varepsilon / T)]$ denotes the Fermi-Dirac distribution function. The functions $K_{\sigma}^{R,(n, m)}(\omega)$ are retarded function corresponding to the following Matsubara function

$$
K_{\sigma}^{(n, m)}(i \omega)=T \sum_{\Omega} \frac{[\tilde{\alpha}(i \Omega+i \omega)-\tilde{\alpha}(i \Omega)]^{n}}{(i \omega)^{n}(i \Omega+\Delta \sigma)^{m}} .
$$

\section{The diagrams IIa and IIb}

The contribution from the diagram IIa to the polarization operator can be written as

$$
\begin{aligned}
& \Pi_{s, \mathrm{pf}}^{(2), \mathrm{IIa}}\left(i \omega_{n}\right)=-\frac{T^{3}}{4} \sum_{\sigma= \pm} \sigma^{2} \sum_{\varepsilon, \Omega, \Omega^{\prime}} \tilde{\alpha}(i \Omega) \tilde{\alpha}\left(i \Omega^{\prime}\right) G_{\sigma}^{2}(i \varepsilon) \\
& \times G_{\sigma}\left(i \varepsilon+i \omega_{n}\right) G_{-\sigma}^{2}(i \varepsilon+i \Omega) G_{\sigma}\left(i \varepsilon+i \Omega+i \Omega^{\prime}\right) .
\end{aligned}
$$

The contribution from the diagram IIb can be found from the expression above by reverting the sign of $\omega_{n}$ : $\Pi_{s, \mathrm{pf}}^{(2), \mathrm{IIb}}\left(i \omega_{n}\right)=\Pi_{s, \mathrm{pf}}^{(2), \mathrm{IIa}}\left(-i \omega_{n}\right)$. Evaluating the sum over the fermionic energy $\varepsilon$, combining two contributions together, and performing analytic continuation, we find

$$
\begin{gathered}
\operatorname{Im} \Pi_{s, \mathrm{pf}}^{R,(2), \operatorname{II}}(\omega)=-\partial_{\Delta} \sum_{\sigma= \pm} \frac{f_{\sigma}-f_{-\sigma}}{2 \omega \sigma} Y_{\sigma} \operatorname{Im} K_{\sigma}^{R,(1,1)}(\omega) \\
+\frac{T}{8} \sum_{\sigma= \pm}\left(f_{\sigma}^{\prime}+f_{-\sigma}^{\prime}\right) \operatorname{Im} K_{\sigma}^{R,(2,2)}(\omega) .
\end{gathered}
$$

Here we introduce the following function

$$
Y_{\sigma}=T \sum_{\Omega} \frac{\tilde{\alpha}(i \Omega)}{i \Omega+\Delta \sigma}
$$


Strictly speaking the sum defining $Y_{\sigma}$ is divergent. The summation is truncated at $\Omega=\Omega_{\max } \sim E_{c}$ which is the model cut-off. The function $Y_{\sigma}$ is therefore cut-off dependent. It is also important to note that when evaluating summation over boson frequencies $\Omega^{\prime}$ and coming across divergent expressions we assume symmetric limits $-\Omega_{\max }<\Omega^{\prime}<\Omega_{\max }$. Truncated symmetric sums allows us to repeatedly shift a summation variable safely.

\section{The diagrams IIIa and IIIb}

The contribution from the diagram IIIa to the polarization operator can be written as

$$
\begin{gathered}
\Pi_{s, \mathrm{pf}}^{(2) \text {,IIIa }}\left(i \omega_{n}\right)=-\frac{T^{3}}{4} \sum_{\sigma= \pm} \sigma^{2} \sum_{\varepsilon, \Omega, \Omega^{\prime}} \tilde{\alpha}(i \Omega) \tilde{\alpha}\left(i \Omega^{\prime}\right) G_{\sigma}^{3}(i \varepsilon) \\
\times G_{\sigma}\left(i \varepsilon+i \omega_{n}\right) G_{-\sigma}(i \varepsilon+i \Omega) G_{-\sigma}\left(i \varepsilon+i \Omega^{\prime}\right) .
\end{gathered}
$$

The contribution from the diagram IIIb can be found from the expression above by reverting the sign of $\omega_{n}$ : $\Pi_{s, \mathrm{pf}}^{(2), \mathrm{IIIb}}\left(i \omega_{n}\right)=\Pi_{s, \mathrm{pf}}^{(2), \mathrm{IIIa}}\left(-i \omega_{n}\right)$. Evaluating the sum over the fermionic energy $\varepsilon$, combining two contributions together, and performing analytic continuation, we find

$$
\begin{gathered}
\operatorname{Im} \Pi_{s, \mathrm{pf}}^{R,(2), \operatorname{III}}(\omega)=\sum_{\sigma= \pm} \frac{f_{\sigma}-f_{-\sigma}}{2 \omega} \operatorname{Im}\left[K_{\sigma}^{R,(1,1)}(\omega)\right]^{2} \\
+\sum_{\sigma= \pm} \frac{f_{\sigma}-f_{-\sigma}}{\omega^{2}} Y_{\sigma} \operatorname{Im} K_{\sigma}^{R,(1,1)}(\omega)
\end{gathered}
$$

\section{The diagrams IVa, IVb, IVc, and IVd}

The contribution from the diagram IVa to the polarization operator can be written as

$$
\begin{aligned}
\Pi_{s, \mathrm{pf}}^{(2), \mathrm{IVa}}\left(i \omega_{n}\right)= & -\frac{T^{3}}{4} \sum_{\sigma= \pm}\left(-\sigma^{2}\right) \sum_{\varepsilon, \Omega, \Omega^{\prime}} \tilde{\alpha}(i \Omega) \tilde{\alpha}\left(i \Omega^{\prime}\right) G_{\sigma}^{2}(i \varepsilon) \\
\times G_{\sigma}(i \varepsilon+ & \left.i \omega_{n}\right) G_{-\sigma}\left(i \varepsilon+i \Omega^{\prime}\right) G_{-\sigma}(i \varepsilon+i \Omega) \\
& \times G_{-\sigma}\left(i \varepsilon+i \Omega+i \omega_{n}\right) .
\end{aligned}
$$

The contribution from the other three diagrams can be found from the expression above by reverting the sign of $\omega_{n}$ and the sign of $\sigma$ and the summation sign: $\Pi_{s, \mathrm{pf}}^{(2), \mathrm{IVb}}\left(i \omega_{n}, \sigma\right)=\Pi_{s, \mathrm{pf}}^{(2), \mathrm{IVa}}\left(-i \omega_{n},-\sigma\right)$, $\Pi_{s, \mathrm{pf}}^{(2), \mathrm{IVc}}\left(i \omega_{n}, \sigma\right)=\Pi_{s, \mathrm{pf}}^{(2), \mathrm{IVa}}\left(i \omega_{n},-\sigma\right), \quad$ and $\Pi_{s, \mathrm{pf}}^{(2), \mathrm{IVd}}\left(i \omega_{n}, \sigma\right)=\Pi_{s, \mathrm{pf}}^{(2), \mathrm{IVa}}\left(-i \omega_{n}, \sigma\right) . \quad$ Evaluating the sum over the fermionic energy $\varepsilon$, combining all four contributions together, and performing analytic
TABLE I. The first few integrals $I_{k}$.

$$
\begin{aligned}
& I_{1}=-\omega\left(\omega^{2}+4 \pi^{2} T^{2}\right) / 3 \\
& I_{2}=\omega^{2}\left(\omega^{2}+4 \pi^{2} T^{2}\right) / 6 \\
& I_{3}=-\omega\left(3 \omega^{4}+20 \pi^{2} T^{2} \omega^{2}+32 \pi^{4} T^{4}\right) / 30 \\
& I_{4}=\omega^{2}\left(\omega^{4}+10 \pi^{2} T^{2} \omega^{2}+24 \pi^{4} T^{4}\right) / 15
\end{aligned}
$$

continuation, we find

$$
\begin{gathered}
\operatorname{Im} \Pi_{s, \mathrm{pf}}^{R,(2), \operatorname{IV}}(\omega)=-\partial_{\Delta} \sum_{\sigma= \pm} \frac{f_{\sigma}-f_{-\sigma}}{\omega \sigma} Y_{\sigma} \operatorname{Im} K_{\sigma}^{R,(1,1)}(\omega) \\
+\sum_{\sigma= \pm} \frac{f_{\sigma}-f_{-\sigma}}{\omega} \operatorname{Im}\left[K_{\sigma}^{R,(1,1)}(\omega)\right]^{2} \\
-T \sum_{\sigma= \pm} \frac{f_{\sigma}^{\prime}+f_{-\sigma}^{\prime}}{4 \omega} \operatorname{Im}\left[2 K_{\sigma}^{R,(2,1)}(\omega)-\omega K_{\sigma}^{R,(2,2)}(\omega)\right] .
\end{gathered}
$$

\section{The diagram $\mathrm{V}$}

The contribution from the diagram $\mathrm{V}$ to the polarization operator can be written as

$$
\begin{aligned}
& \Pi_{s, \mathrm{pf}}^{(2), \mathrm{V}}\left(i \omega_{n}\right)=-\frac{T^{3}}{4} \sum_{\sigma= \pm} \sigma^{2} \sum_{\varepsilon, \Omega, \Omega^{\prime}} \tilde{\alpha}(i \Omega) \tilde{\alpha}\left(i \Omega^{\prime}\right) G_{\sigma}^{2}(i \varepsilon) \\
& \times G_{\sigma}^{2}\left(i \varepsilon+i \omega_{n}\right) G_{-\sigma}\left(i \varepsilon+i \Omega^{\prime}\right) G_{-\sigma}(i \varepsilon+i \Omega) .
\end{aligned}
$$

Evaluating the sum over the fermionic energy $\varepsilon$ and performing analytic continuation, we find

$$
\begin{gathered}
\operatorname{Im} \Pi_{s, \mathrm{pf}}^{R,(2), \mathrm{V}}(\omega)=-\sum_{\sigma= \pm} \frac{f_{\sigma}-f_{-\sigma}}{\omega^{2}} Y_{\sigma} \operatorname{Im} K_{\sigma}^{R,(1,1)}(\omega) \\
-\partial_{\Delta} \sum_{\sigma= \pm} \frac{f_{\sigma}-f_{-\sigma}}{2 \omega \sigma} Y_{\sigma} \operatorname{Im} K_{\sigma}^{R,(1,1)}(\omega) \\
-T \sum_{\sigma= \pm} \frac{f_{\sigma}^{\prime}+f_{-\sigma}^{\prime}}{8 \omega} \operatorname{Im}\left[2 K_{\sigma}^{R,(2,1)}(\omega)-\omega K_{\sigma}^{R,(2,2)}(\omega)\right] .
\end{gathered}
$$

\section{The result for $\operatorname{Im} \Pi_{s, \mathrm{pf}}^{R}(\omega)$ in the $2 \mathrm{~d}$ order in $g$}

Combining all contributions, Eqs. A2, A5, A8, A10, and (A12, together, we find

$$
\begin{gathered}
\operatorname{Im} \Pi_{s, \mathrm{pf}}^{R,(2)}(\omega)=\frac{T}{2} \sum_{\sigma}\left(f_{\sigma}^{\prime}+f_{-\sigma}^{\prime}\right) \operatorname{Im} K_{\sigma}^{R,(2,2)}(\omega) \\
-2 \partial_{\Delta} \sum_{\sigma= \pm} \frac{f_{\sigma}-f_{-\sigma}}{\omega \sigma} Y_{\sigma} \operatorname{Im} K_{\sigma}^{R,(1,1)}(\omega) \\
\quad-T \sum_{\sigma} \frac{f_{\sigma}^{\prime}+f_{-\sigma}^{\prime}}{\omega} \operatorname{Im} K_{\sigma}^{R,(2,1)}(\omega) \\
+2 \sum_{\sigma= \pm} \frac{f_{\sigma}-f_{-\sigma}}{\omega} \operatorname{Im}\left[K_{\sigma}^{R,(1,1)}(\omega)\right]^{2}
\end{gathered}
$$


The following comment is in order here. Alternatively, one can compute the admittance by means of the currentcurrent correlation function [7. The latter consists of two operators which are of the first and second order in $g$ [50]. Then the first and second lines in Eq. A14 comes from the renormalization of the operator of the first order in $g$ whereas the third and forth lines correspond to the contributions from the operator of the second order in $g$. Using Eq. 26, we find

$$
\begin{gathered}
\operatorname{Im} \Pi_{s}^{R,(2)}(\omega)=-2 \tanh \frac{\beta \Delta}{2} \sum_{\sigma= \pm} \frac{\sigma}{\omega} \operatorname{Im}\left[K_{\sigma}^{R,(1,1)}(\omega)\right]^{2} \\
+\frac{2}{\cosh (\beta \Delta / 2)} \partial_{\Delta} \sum_{\sigma= \pm} \frac{\sinh (\beta \Delta / 2)}{\omega} Y_{\sigma} \operatorname{Im} K_{\sigma}^{R,(1,1)}(\omega) \\
\quad+\sum_{\sigma} \operatorname{Im}\left[\frac{1}{2} K_{\sigma}^{R,(2,2)}-\frac{1}{\omega} K_{\sigma}^{R,(2,1)}(\omega)\right] .
\end{gathered}
$$

This expression is convenient for analysis at $|\omega|, T \ll|\Delta|$. Converting the sum over Matsubara frequencies in Eq. (A3) into the integral and performing analytic continuation, $i \omega \rightarrow \omega+i 0$, we obtain

$$
\begin{aligned}
\operatorname{Im} K_{\sigma}^{R,(1,1)}(\omega) & =\int_{-\infty}^{\infty} \frac{d \varepsilon}{2 \pi \omega}\left(\mathcal{B}_{\varepsilon}-\mathcal{B}_{\varepsilon+\omega}\right) \operatorname{Im} D_{\sigma}^{R}(\varepsilon) \\
& \times \operatorname{Im} \tilde{\alpha}^{R}(\varepsilon+\omega)
\end{aligned}
$$

Here $\mathcal{B}_{\varepsilon}=\operatorname{coth}(\varepsilon / 2 T), \tilde{\alpha}^{R}(\omega)=-\tilde{\alpha}^{A}(\omega)=-i g \omega /(4 \pi)$, and $D_{\sigma}^{R}(\varepsilon)=[\varepsilon+\Delta \sigma+i 0]^{-1}$. Since $\operatorname{Im} D_{\sigma}^{R}(\varepsilon)=$ $-\pi \delta(\varepsilon+\Delta \sigma)$, the imaginary part of $K_{\sigma}^{R,(1,1)}$ is exponentially small, $\sim \exp (-|\Delta| / T)$. We note that $K_{\sigma}^{R,(1,1)}$ is defined by the divergent Matsubara sum. It should be understood as a finite sum truncated at $\Omega=\Omega_{\max } \sim E_{c}$. Then the analytical continuation is possible. Moreover the imaginary part of $K_{\sigma}^{R,(1,1)}$ is $E_{c}$ independent.

The terms proportional to $K_{\sigma}^{R,(1,1)}$ are responsible for the renormalization of the first order perturbative result (27). Therefore, only the last line in Eq. A14 contributes to $\operatorname{Im} \Pi_{s}^{R,(2)}(\omega)$ in the regime $|\omega|, T \ll|\Delta|$.
Again converting the sun over Matsubara frequencies in Eq. A3 into the integral and performing analytic continuation, $i \omega \rightarrow \omega+i 0$, we find

$$
\begin{aligned}
& \operatorname{Im} K_{\sigma}^{R,(2,1)}(\omega)=\int_{-\infty}^{\infty} \frac{d \varepsilon}{\pi \omega^{2}}\left(\mathcal{B}_{\varepsilon+\omega}-\mathcal{B}_{\varepsilon}\right) \operatorname{Re} D_{\sigma}^{R}(\varepsilon) \operatorname{Im} \tilde{\alpha}^{R}(\varepsilon) \\
& \quad \times \operatorname{Im} \tilde{\alpha}^{R}(\varepsilon+\omega)=\left(\frac{g}{4 \pi}\right)^{2} \sum_{k=1}^{\infty} \frac{(-1)^{k-1} I_{k}}{\pi \omega^{2}(\Delta \sigma)^{k}} .
\end{aligned}
$$

Here we perform expansion in series in $1 / \Delta$. The functions $I_{k}$ are defined as follows

$$
I_{k}=\int_{-\infty}^{\infty} d \varepsilon \varepsilon^{k}(\varepsilon+\omega)\left(\mathcal{B}_{\varepsilon+\omega}-\mathcal{B}_{\varepsilon}\right) .
$$

We list several first functions $I_{k}$ (for $k=1,2,3,4$ ) in Table I. We note that the very same functions $I_{k}$ determine the imaginary part of $K_{\sigma}^{R,(2,2)}(\omega)$ :

$$
\begin{gathered}
\operatorname{Im} K_{\sigma}^{R,(2,2)}(\omega)=-\sigma \partial_{\Delta} \operatorname{Im} K_{\sigma}^{R,(2,2)}(\omega) \\
=\left(\frac{g}{4 \pi}\right)^{2} \sum_{k=1}^{\infty} \frac{k(-1)^{k-1} I_{k}}{\pi \omega^{2}(\Delta \sigma)^{k+1}}
\end{gathered}
$$

Using the functions $I_{k}$ from the Table I, from Eq. A14 we obtain the result 30 in the main text.

Finally, we note that for the case of $q=k$, i.e. at the Coulomb peaks, one cannot adopt the pseudofermion technique and has to take into account all charging states. Then, the contribution to $\operatorname{Re} \mathcal{G}(\omega)$ due to the inelastic cotunneling is given by the last line of Eq. A14 multiplied by a factor of 2 and with the following substitutions: $\Delta \rightarrow E_{c}$ and

$$
\frac{1}{\omega} \rightarrow \frac{1}{\omega}\left(1+\frac{\omega^{2}}{2 E_{c}\left(2 E_{c}-\omega \sigma\right)}\right) .
$$

It is due to this additional term the contribution of the order of $1 / E_{c}^{4}$ cancels and the admittance becomes proportional to $1 / E_{c}^{6}[7]$.
${ }^{1}$ G. Schön and A. Zaikin, "Quantum coherent effects, phase transitions, and the dissipative dynamics of ultra small tunnel junctions," Phys. Rep. 198, 237 (1990).

2 Z. Phys. B: Condens. Matter 85, 317 (1991, special issue on single charge tunneling, ed. by H. Grabert and H. Horner.).

3 H. Grabert and M. H. Devoret, eds., Single Charge Tunneling (Plenum, New York, 1992).

${ }^{4}$ Y. Blanter and M. Büttiker, "Shot noise in mesoscopic conductors," Phys. Rep. 336, 1 (2000)

5 I. Aleiner, P. Brouwer, and L. Glazman, "Quantum effects in Coulomb blockade," Phys. Rep. 358, 309 (2002).

${ }^{6}$ L. I. Glazman and M. Pustilnik, "Low-temperature transport through a quantum dot," in New Directions in Mesoscopic Physics (Towards to Nanoscience), edited by
R. Fazio, V. F. Gantmakher, and Y. Imry (Kluwer, Dordrecht, 2003).

7 Yu. V. Nazarov, "Dissipation in a Coulomb capacitor," Sov. J. Low Temp. Phys. 16, 422 (1990).

${ }^{8}$ K. A. Matveev, "Quantum fluctuations of the charge of a metal particle under the Coulomb blockade conditions," Sov. Phys. JETP 72, 892 (1991).

${ }^{9}$ H. Grabert, "Rounding of the Coulomb staircase by the tunneling conductance," Physica B 194, 1011 (1994).

$10 \mathrm{H}$. Grabert, "Charge fluctuations in the single-electron box: Perturbation expansion in the tunneling conductance," Phys. Rev. B 50, 17364 (1994).

11 K. A. Matveev, "Coulomb blockade at almost perfect transmission," Phys. Rev. B 51, 1743 (1995). 
${ }^{12} \mathrm{X}$. Wang and H. Grabert, "Coulomb charging at large conduction," Phys. Rev. B 53, 12621 (1996)

13 G. Göppert, H. Grabert, N. V. Prokof'ev, and B. V. Svistunov, "Effect of tunneling conductance on the Coulomb staircase," Phys. Rev. Lett. 81, 2324 (1998)

14 I. S. Beloborodov, A. V. Andreev, and A. I. Larkin, "Twoloop approximation in the Coulomb blockade problem," Phys. Rev. B 68, 024204 (2003).

15 M. Büttiker, H. Thomas, and A. Pretre, "Mesoscopic capacitors," Phys. Lett. A 180, 364 (1993)

16 M. Büttiker and A. M. Martin, "Charge relaxation and dephasing in Coulomb-coupled conductors," Phys. Rev. B 61, 2737 (2000)

S. E. Nigg, R. López, and M. Büttiker, "Mesoscopic charge relaxation," Phys. Rev. Lett. 97, 206804 (2006).

18 S. E. Nigg and M. Büttiker, "Quantum to classical transition of the charge relaxation resistance of a mesoscopic capacitor," Phys. Rev. B 77, 085312 (2008).

19 Z. Ringel, Y. Imry, and O. Entin-Wohlman, "Delayed currents and interaction effects in mesoscopic capacitors," Phys. Rev. B 78, 165304 (2008)

20 H. C. Park and K.-H. Ahn, "Admittance and noise in an electrically driven nanostructure: Interplay between quantum coherence and statistics," Phys. Rev. Lett. 101, $116804(2008)$

${ }^{21}$ J. Gabelli, G. Feve, J.-M. Berroir, B. Plaçais, A. Cavanna, B. Etienne, Y. Jin, and D. C. Glattli, "Violation of Kirchhoff's laws for a coherent $R C$ circuit," Science 313, 499 (2006)

${ }^{22}$ F. Persson, C. M. Wilson, M. Sandberg, G. Johansson, and P. Delsing, "Excess dissipation in a dingle-electron box: The Sisyphus resistance," Nano Lett. 10, 953-957 (2010)

¿3 C. Ciccarelli and A. J. Ferguson, "Impedance of the singleelectron transistor at radio-frequencies," New J. Phys. 13, 093015 (2011)

${ }^{24}$ S. J. Chorley, J. Wabnig, Z. V. Penfold-Fitch, K. D. Petersson, J. Frake, C. G. Smith, and M. R. Buitelaar, "Measuring the complex admittance of a carbon nanotube double quantum dot," Phys. Rev. Lett. 108, 036802 (2012)

25 J. C. Frake, S. Kano, C. Ciccarelli, J. Griffiths, M. Sakamoto, T. Teranishi, Y. Majima, C. G. Smith, and M. R. Buitelaar, "Radio-frequency capacitance spectroscopy of metallic nanoparticles," Sci. Rep. 5, 10858 (2015)

20 C. Mora and K. Le Hur, "Universal resistances of the quantum resistance-capacitance circuit," Nat. Phys. 6, 697 (2010).

27 J. Korringa, "Nuclear magnetic relaxation and resonance line shift in metals," Physica 16, 601 (1950).

${ }^{28} \mathrm{H}$. Shiba, "The Korringa relation for the impurity nuclear spin-lattice relaxation in dilute Kondo alloys," Prog. Theor. Phys. 54, 967 (1975).

29 M. Filippone and C. Mora, "Fermi liquid approach to the quantum $R C$ circuit: Renormalization group analysis of the Anderson and Coulomb blockade models," Phys. Rev. B 86, 125311 (2012)

${ }^{30}$ P. Dutt, T. L. Schmidt, C. Mora, and K. Le Hur, "Strongly correlated dynamics in multichannel quantum $R C$ cir- cuits," Phys. Rev. B 87, 155134 (2013)

31 Ya. I. Rodionov, I. S. Burmistrov, and A. S. Ioselevich, "Charge relaxation resistance in the Coulomb blockade problem," Phys. Rev. B 80, 035332 (2009).

32 I. S. Burmistrov and A. M. M. Pruisken, "Coulomb blockade and superuniversality of the $\theta$ angle," Phys. Rev. Lett. 101, 056801 (2008)

33 I. S. Burmistrov and A. M. M. Pruisken, "Macroscopic charge quantization in single-electron devices," Phys. Rev. B 81, 085428 (2010).

${ }^{34}$ A. G. Semenov, "On the macroscopic quantization in mesoscopic rings and single-electron devices," (2013), arXiv:1307.6615

35 D. V. Averin and Yu. V. Nazarov, "Virtual electron diffusion during quantum tunneling of the electric charge," Phys. Rev. Lett. 65, 2446 (1990)

36 F. Mezei, "Theory of electron tunneling via real intermediate states," Phys. Rev. B 4, 3775 (1971)

37 R. I. Shekhter, "Zero anomalies in the resistance of a tunnel junction containing metallic inclusions in the oxide layer," Sov. Phys. JETP 36, 747 (1973).

38 I. O. Kulik and R. I. Shekhter, "Kinetic phenomena and charge discreteness effects in granulated media," Sov. Phys. JETP 41, 308 (1975).

39 A. A. Abrikosov, L. P. Gorkov, and I. E. Dzyaloshinski, Methods of Quantum Field Theory in Statistical Physics (Prentice-Hall, Englewood Cliffs, NJ, 1963).

40 A. A. Abrikosov, "Electron scattering on magnetic impurities in metals and anomalous resistivity effects," Physics. Physique. Fizika. 2, 5 (1965).

41 A. I. Larkin and V. I. Melnikov, "Magnetic impurities in an almost magnetic metal," Sov. Phys. JETP 34, 656 (1972).

42 S. Sachdev and J. Ye, "Gapless spin-fluid ground state in a random quantum Heisenberg magnet," Phys. Rev. Lett. 70, 3339 (1993)

${ }^{43}$ L. Zhu and Q. Si, "Critical local-moment fluctuations in the Bose-Fermi Kondo model," Phys. Rev. B 66, 024426 (2002)

${ }^{44}$ G. Zaránd and E. Demler, "Quantum phase transitions in the Bose-Fermi Kondo model," Phys. Rev. B 66, 024427 (2002)

45 H. Schoeller and G. Schön, "Mesoscopic quantum transport: Resonant tunneling in the presence of a strong Coulomb interaction," Phys. Rev. B 50, 18436 (1994)

${ }^{46}$ G. Zaránd, G. T. Zimányi, and F. Wilhelm, "Twochannel versus infinite-channel Kondo models for the single-electron transistor," Phys. Rev. B 62, 8137 (2000)

47 Ya. I. Rodionov, I. S. Burmistrov, and N. M. Chtchelkatchev, "Relaxation dynamics of the electron distribution in the Coulomb-blockade problem," Phys. Rev. B 82, 155317 (2010).

48 Ya. I. Rodionov and I. S. Burmistrov, "Out-of-equilibrium admittance of single electron box under strong Coulomb blockade," JETP Lett. 92, 696 (2010).

49 Ya. I. Rodionov and I. S. Burmistrov, unpublished.

50 Eshel Ben-Jacob, Emil Mottola, and Gerd Schön, "Quantum shot noise in tunnel junctions," Phys. Rev. Lett. 51, 2064 (1983). 